ACT A C H E I CA SCA N D N A V I CA 20 (1966) $2368-2362$

\title{
A Disaccharide Dibenzoate from Daviesia latifolia
}

\author{
BERTIL HANSSON, INGVAR JOHANSSON and BENGT LINDBERG
}

\author{
Institutionen för organisk kemi, Stockholms Universitet, Stockholm and \\ Institutionen för träkemi, Kungl. Tekniska Högskolan, Stockholm, Sweden
}

\begin{abstract}
The disaccharide dibenzoate, previously isolated from the leaves of Daviesia latifolia, ${ }^{1}$ and believed to be an ester of a D-glucosyl-Dxyloside, has been reinvestigated. The disaccharide is a 2-O-D-apiosylD-glucose, having the same structure as the disaccharide moiety of apiin. One of the benzoate ester groups is situated at C-1 of the Dglucose residue, the other at the primary position of the D-apiose residue.
\end{abstract}

The Australian shrub Daviesia latifolia (Leguminosae) is known as the 1 "native' hop bush" because of the bitter taste of the leaves. The bitter principle was isolated as early as 1898 by Bosisto ${ }^{2}$ and was studied more closely by Power and Salway. 1 The latter authors found that it was a dibenzoate of a disaccharide. One of the sugar components was identified as D-glucose. The other sugar was claimed to be D-xylose. Analytical results showed it to be a pentose, the optical rotation of a hydrolysate was in reasonably good agreement with the estimated value and the pentose gave a phenylosazone of m.p. $157-158^{\circ}$. The corresponding $D$-xylose derivative melts at $160-162^{\circ}$. From the fact that the disaccharide did not reduce Fehling's solution the conclusion was drawn that it was a D-glucosyl-D-xyloside, with no free reducing group.

The structure of the disaccharide dibenzoate has now been reinvestigated. The substance had m.p. $148-150^{\circ},[\alpha]_{\mathrm{D}}{ }^{22}-106^{\circ}[\alpha]_{578}{ }^{22}-112^{\circ}$ (methanol) and gave a pentaacetate, m.p. $203-204^{\circ},[\alpha]_{578}{ }^{22}-35^{\circ}$ (chloroform), in good agreement with previously recorded values. The acetylation did not go to completion, and a tetraacetate, m.p. $168-170^{\circ},[\alpha]_{578}{ }^{22}-64^{\circ}$, was also obtained. Alkaline hydrolysis yielded benzoic acid and the disaccharide, $[\alpha]_{578}^{22}-51^{\circ}$ (water). The disaccharide on acid hydrolysis yielded D-glucose and a sugar which had a higher $R_{F}$-value than $\mathrm{D}$-xylose and gave a different colour reaction with the $p$-anisidine spray reagent. This sugar, which was paper chromatographically indistinguishable from $\mathbf{D}$-apiose, was isolated by preparative paper chromatography and converted into the crystalline isopropylidene derivative, m.p. $81-82^{\circ},[\alpha]_{578}{ }^{22}-61^{\circ}$, indistinguishable from an authentic sample of di-O-isopropylidene-D-apiose. The phenylosazone of D-apiose melts at 155 $157^{\circ}$, rather close to that of $\mathrm{D}$-xylose, which explains the mistake of the earlier investigators. 
The free disaccharide, but not the dibenzoate, gave a positive reaction with the anisidine reagent, indicating that it was a reducing disaccharide, with one of the $O$-benzoyl groups in the C-1 position. After borohydride reduction and acid hydrolysis the disaccharide yielded apiose and glucitol, showing that it is an apiosyl-glucose. The negative reaction with Fehling's solution, observed by Power and Salway, must be reinterpreted and indicates that the glucose residue is substituted in the 2-position.

On methylation and acid hydrolysis the disaccharide yielded two methylated sugars, chromatographically (paper and thin layer) indistinguishable from 3,4,6-tri- $O$-methyl-D-glucose and tri- $O$-methyl-D-apiose, obtained by similar treatment of apiin. The trimethylsilyl ethers prepared from the tri-O-methyl$D$-glucose were indistinguishable, by GLC, from those prepared from apiin but different from those given by $2,3,4-, 2,3,6-$, or 2,4,6-tri- $O$-methyl-D-glucose. The tri- $O$-methyl-D-glucose further gave a phenylosazone, m.p. 80-81 ${ }^{\circ}$, indistinguishable from an authentic sample.

$\mathrm{D}$-Apiose, on methylation and glycosidation, gives four isomeric methyl tri- $O$-methyl-D-apiosides. ${ }^{3}$ Only two of these are obtained from fully methylated apiin, and Jones and coworkers ${ }^{3,4}$ have recently shown, by unambigous synthesis, that in these the two methoxyls at C-2 and C-3 are in the cis-position. The tri- $O$-methyl-D-apiose obtained from the Daviesia disaccharide also gave two methyl glycosides, indistinguishable, by GLC, from those obtained from apiin. This, together with the low optical rotation of the Daviesia disaccharide, shows that it has the same structure, 2-O-(D-apio-D-furanosyl)-Dglucose (I), as the disaccharide moiety of apiin, presumably with a $\beta$-D-apiosyl linkage.

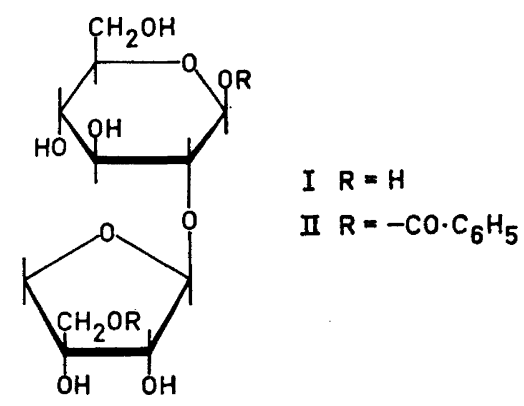

In order to locate the $O$-benzoate groups in the natural product, it was subjected to partial acid hydrolysis. The hydrolysate was separated by preparative paper chromatography to give six components. Three of these were identified as D-glucose, D-apiose, and unchanged starting material, respectively. $D$-Glucose was formed rapidly, D-apiose more slowly. One of the other products did not react with the $p$-anisidine spray reagent but yielded glucose after alkaline hydrolysis, indicating that it was 1-O-benzoyl-D-glucose. A second product, rapidly formed and relatively stable, gave a positive reaction with the $p$-anisidine spray reagent and yielded $\mathrm{D}$-apiose on alkaline hydrolysis. The third component yielded the free disaccharide on alkaline hydrolysis.

Acta Chem. Scand. 20 (1966) No. 9 
These results show that one $O$-benzoate group is located at $\mathrm{C}-1$ of the glucose residue, presumably in $\beta$-position because of the low optical rotation of the natural product, and the other in the $D$-apiose residue. In naturally acylated sugars the $O$-acyl groups are most often found at C-1 or at a primary position.

In agreement with these results the dibenzoate, after methylation with methyl iodide and silver oxide in dimethylformamide, and hydrolysis, yielded 3,4,6-tri- $O$-methyl-D-glucose and a di- $O$-methyl-D-apiose. The latter, on further methylation with the same reagents, yielded a mixture of four methyl tri-Omethyl-D-apiosides, as demonstrated by GLC. The only di- $O$-methyl-ether of $\mathrm{D}$-apiose which should give four isomeric glycosides is the 2,3-di- $O$-methyl ether (III). The experiments therefore prove that the di- $O$-benzoate has the structure(II) with one benzoate ester group at the primary position in the $\mathrm{D}$-apiose residue and the other at $\mathrm{C}-1$ of the $\mathrm{D}$-glucose residue.

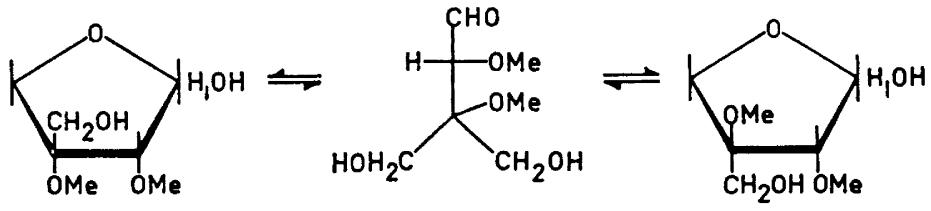

III

Apiose has a high paper electrophoretic mobility $\left(M_{\mathrm{G}}=43\right)$ in sulphonated phenyl boronic acid ${ }^{5}$ at $\mathrm{pH}$ 6.5. This is due to the presence of adjacent cishydroxyls in a 5-membered ring. The high mobility of the dibenzoate $\left(M_{\mathrm{G}}=25\right)$ in the same buffer indicates that the hydroxyls at C-2 and C-3 in the D-apiose residue are unsubstituted and in the cis-position, in agreement with the assigned structure.

On lead tetraacetate oxidation, the dibenzoate consumed one mole of oxidant very rapidly, followed by a much slower consumption of reagent. This is also in agreement with the assigned structure, as the cis-hydroxyl grouping in the $\mathrm{D}$-apiose residue should be highly reactive. The results of periodate oxidation were inconsistant probably due to simultaneous hydrolysis of ester groups.

The NMR spectrum of the fully acetylated natural product showed, inter alia, a doublet ( $\tau=3.95 \mathrm{ppm}, J_{1,2}=8.0 \mathrm{cps}$ ) which could be assigned to H-1 in the glucopyranose residue and confirms its $\beta$-configuration. The same doublet was also observed in the tetraacetate, which also showed absorption in IR at $3490 \mathrm{~cm}^{-1}$. It is reasonable to assume that the tertiary hydroxyl group, in the apiosyl residue, is the group resistant to acetylation.

In the NMR spectrum of the fully trimethylsilylated disaccharide dibenzoate, the signal from the $C-1$ proton of the $D$-apiose residue $(\tau=4.82 \mathrm{ppm}$, $J_{1,2}=2.0 \mathrm{cps}$ ) was well separated from the other signals, which, with the exception of those from the aromatic protons and the C-1 proton of the Dglucose residue, appeared at higher fields. The corresponding signal from fully trimethylsilylated phenyl $\beta$-D-xylofuranoside appeared at $\tau=4.68 \mathrm{ppm}$, $J_{1,2}=2.2 \mathrm{cps}$. A higher coupling constant $\left(J_{1,2}=3.9\right)$ was observed for $1,3,5$ - 
tri-O-benzoyl- $\alpha$-D-ribofuranose, ${ }^{6}$ with a cis-relationship between the $\mathrm{C}-1$ and C-2 protons. These results therefore support the assumed $\beta$-configuration of the $\mathrm{D}$-apiose residue in the disaccharide.

\section{EXPERIMENTAL}

Paper chromatography was carried out on Whatman No. 1 and No. 3 MM filter papers, using the following solvent systems: (a) butanone, saturated with water; (b) butan-1-olacetone-water (4:5:1); (c) ethyl acetate-acetic acid-water (3:1:1); (d) butan-1-ol-ethanolbenzene-water (10:3:5:3). The separated materials were detected with $p$-anisidine hydrochloride in butan-1-ol or with the silver nitrate-sodium hydroxide reagent. Thin layer chromatography was carried out on silica gel $\mathrm{GF}_{254}$, using as solvent toluene-ethyl ether (1:2) and detection effected under UV-light or with M sulfuric acid, followed by heating. GLC was carried out on a Perkin-Elmer 800 gas chromatograph, using either (a) a BDS column at $130^{\circ}$ or (b) a Carbowax $20 \mathrm{M}$ column at $140^{\circ}$. Paper electrophoresis was carried out in $0.05 \mathrm{M}$ sulphonated phenyl boronic acid of $\mathrm{pH} 6.5$, at $40^{\circ}$, on Whatman 3MM filter paper. Lead tetraacetate oxidations were carried out in the presence of potassium acetate as described by Charlsson and Perlin. ${ }^{7}$ NMR spectra were measured in deuterochloroform on a Varian A60 spectrometer. Melting points are corrected. Solutions were concentrated under reduced pressure below $40^{\circ}$.

Isolation of the disaccharide dibenzoate. Dried milled leaves of Daviesia latifolia $(500 \mathrm{~g})$ were continuously extracted with ether for $6 \mathrm{~h}$, to remove most of the lipophilic material. The extraction was then continued with fresh ether for 6 days. This extract was concentrated to $250 \mathrm{ml}$ and extracted with water $(4 \times 250 \mathrm{ml})$. On concentration of the aqueous extract, the disaccharide dibenzoate crystallised. Crystallisation, first from ethyl acetate and then from water, yielded the pure substance $(2.0 \mathrm{~g}), \mathrm{m} . \mathrm{p} .148-150^{\circ},[\alpha]_{\mathrm{D}}{ }^{22}-106^{\circ}$, $[\alpha]_{578}{ }^{22}-112^{\circ}$ (c 1.0, methanol).

Part of the dibenzoate $(0.48 \mathrm{~g})$ was acetylated with acetic anhydride in pyridine, and the pentaacetate $(100 \mathrm{mg})$, m.p. $203-204^{\circ},[\alpha]_{678^{22}}-35^{\circ}$ (c 1.0, chloroform), was obtained after repeated crystallisations from ethanol. The mother liquors contained the tetraacetate, which was obtained in a pure state $(210 \mathrm{mg}), \mathrm{m} . \mathrm{p} .168-170^{\circ},[\alpha]_{578^{22}}-64^{\circ}$ (c 1.0, chloroform), by preparative TLC and crystallisations from ethanol. (Found: C 57.2; $\mathrm{H} \mathrm{5.26} ; \mathrm{C}_{33} \mathrm{H}_{36} \mathrm{O}_{13}$ requires: $\mathrm{C} \mathrm{57.6;} \mathrm{H}$ 5.27). Acetylation of the tetraacetate, in the same manner, yielded a mixture of the two components.

Acid hydrolysis of the disaccharide dibenzoate. The natural product $(200 \mathrm{mg})$ was hydrolysed in $M$ aqueous sulphuric acid at $100^{\circ}$ overnight, neutralised (Dowex 3, free base) and concentrated. Paper chromatography (solvents a, b, and c) revealed the presence of glucose and apiose. The latter was isolated by thick filter paper chromatography (solvent a) and transferred into the di-O $O$-isopropylidene derivative, as described by Bell. ${ }^{8}$ The di- $O$. isopropylidene-D-apiose $(50 \mathrm{mg})$ melted at $81-82^{\circ}$, undepressed on admixture with an authentic sample, and had $[\alpha]_{578}{ }^{22}+61^{\circ}$ (c 1.0, chloroform).

Isolation of the disaccharide. The dibenzoate $(100 \mathrm{mg})$ was dissolved in hot water $(15 \mathrm{ml})$ and, after cooling to room temperature, $0.3 \mathrm{M}$ sodium hydroxide $(5 \mathrm{ml})$ was added. After $3 \mathrm{~h}$ at room temperature the solution was neutralised (Dowex 50, $\mathbf{H}^{+}$), benzoic acid (m.p. $\left.120-121^{\circ}\right)$ was removed by extraction with ether $(3 \times 15 \mathrm{ml})$ and the solution was concentrated, to give the amorphous disaccharide $(55 \mathrm{mg})$. The disaccharide, after borohydride reduction and acid hydrolysis yielded a mixture of apiose and glucitol, as revealed by paper chromatography (solvents $a, b$, and c).

Partial acid hydrolysis of the disaccharide dibenzoate $(100 \mathrm{mg})$ was performed in 0.005 M sulphuric acid $(10 \mathrm{ml})$ at $100^{\circ}$ for $105 \mathrm{~min}$. The product, after neutralisation (Dowex 3 , free base), was fractionated by thick filter paper chromatography (solvent a) to give 6 components. Three of these were identified as glucose, apiose, and unchanged starting material, respectively. The other three, after treatment with $0.5 \mathrm{M}$ aqueous sodium hydroxide for $5 \mathrm{~h}$ at room temperature, yielded glucose, apiose, and disaccharide, respectively.

Methylation analysis of the disaccharide. The dried disaccharide $(290 \mathrm{mg})$ was methylated in dimethyl formamide $(15 \mathrm{ml})$ with methyl iodide $(5 \mathrm{ml})$ and silver oxide $(2 \mathrm{~g})$ accord. ing to Kuhn's procedure. The methylated product $(230 \mathrm{mg})$ was dissolved in acetic acid $(3 \mathrm{ml}), 2 \mathrm{M}$ hydrochloric acid $(10 \mathrm{ml})$ was added, the solution was kept at $100^{\circ}$ over-

Acta Chem. Scand. 20 (1966) No. 9 
night, cooled, neutralised (Dowex 3, free base) and concentrated to a syrup. This was dissolved in water $(5 \mathrm{ml})$ and extracted with chloroform $(5 \times 5 \mathrm{ml})$. The combined chloroform extracts were dried over sodium sulphate and concentrated to a syrup (105 $\mathrm{mg}$ ), consisting of 2,3,4-tri-O-methyl-D-apiose. Concentration of the aqueous phase yielded 3,4,6-tri- $O$-methyl-D-glucose (115 mg). Authentic samples of these methylated products were prepared from apiin. ${ }^{3}$ The methyl ethers from the disaccharide and those from apiin were indistinguishable on paper chromatography (solvents $b$ and $d$ ). The GLC patterns of their trimethylsilyl ethers ${ }^{10}$ (column a) were indistinguishable, as were also GLC chromatograms of their methyl glycosides (column b). The methyl tri-O-methyl-D-apioside mixture gave two peaks, with the relative retention times, compared to methyl $2,3,4,6$ tetra- $O$-methyl- $\alpha-D-g l u c o s i d e$, of 0.36 and 0.52 . Hulyalkar et al ${ }^{3}$ report 0.343 and 0.483 for the methyl 2,3,4-tri-O-methyl- $\alpha+\beta$-D-apio-D-furanosides on a similar column, at $150^{\circ}$.

Methylation analysis of the disaccharide dibenzoate. The disaccharide dibenzoate (100 mg) was methylated and the methylated product was hydrolysed as described above. The hydrolysate contained two main components, which were separated by paper chromatography (solvent b). One of these $(40 \mathrm{mg}$ ) was indistinguishable from $3,4,6$-tri-O. methyl-D-glucose. The second component $(35 \mathrm{mg})$, on further methylation by the same procedure, followed by treatment with methanolic hydrochloric acid, yielded a product which on GLC gave four peaks (column b), with the retention times, relative to methyl $2,3,4,6$-tetra- $O$-methyl- $\alpha$-D-glucoside, of $0.36,0.51,0.63$, and 0.95 , respectively. The values reported by Hulyalkar et al.$^{3}$ for the four isomeric, fully methylated methyl apiosides on a similar column at $150^{\circ}$ are $0.343,0.483,0.582$, and 0.905 .

Acknowledgements. The authors are indebted to Dr. J. R. Price, Melbourne, for a generous sample of the plant material and to Drs. D. J. Bell, Edinburgh, and D. H. Ball, Natick, for samples of apiin and di-O-isopropylidene-D-apiose, respectively. The NMR spectra were kindly determined by Mr. K.-I. Dahlqvist, Stockholm.

\section{REFERENCES}

1. Power, F. B. and Salway, A. H. J. Chem. Soc. 105 (1914) 767, 1062.

2. Bosisto, J. Pharm. J. 60 (1898) 187.

3. Hulyalkar, R. K., Jones, J. K. N. and Perry, M. B. Can. J. Chem. 43 (1965) 2085.

4. Williams, D. T. and Jones, J. K. N. Can. J. Chem. 42 (1964) 69.

5. Garegg, P. J. and Lindberg, B. Acta Chem. Scand. 15 (1961) 1913.

6. Hall, L. D. Chem. Ind. (London) 1963950.

7. Charlsson, A. J. and Perlin, A. S. Can. J. Chem. 34 (1956) 1200.

8. Bell, D. J. Methods Carbohydrate Chem. 1 (1962) 261.

9. Kuhn, R., Trischmann, H. and Löw, I. Angew. Chem. 67 (1955) 32.

10. Sweeley, C. C., Bentley, R., Makita, M. and Wells, W. W. J. Am. Chem. Soc. 85 (1963) 2497.

Received June 8, 1966. 